\title{
THE SOLUTION OF LENGTH FOUR EQUATIONS OVER GROUPS
}

\author{
MARTIN EDJVET AND JAMES HOWIE
}

\begin{abstract}
Let $G$ be a group, $F$ the free group generated by $t$ and let $r(t) \in$ $G * F$. The equation $r(t)=1$ is said to have a solution over $G$ if it has a solution in some group that contains $G$. This is equivalent to saying that the natural map $G \rightarrow\langle G * F \mid r(t)\rangle$ is injective. There is a conjecture (attributed to $M$. Kervaire and $F$. Laudenbach) that injectivity fails only if the exponent sum of $t$ in $r(t)$ is zero. In this paper we verify this conjecture in the case when the sum of the absolute values of the exponent of $t$ in $r(t)$ is equal to four.
\end{abstract}

\section{INTRODUCTION}

Let $G$ be a group and $t$ an unknown. Then an equation in $t$ over $G$ is an expression

$$
g_{0} t^{e(1)} g_{1} \cdots t^{e(n)} g_{n}=1 \quad\left(g_{i} \in G, e(i)=\mp 1\right),
$$

and a solution to (*) over $G$ is an embedding of $G$ into a group $H$, together with an element $h$ of $H$, such that

$$
g_{0} h^{e(1)} g_{1} \cdots h^{e(n)} g_{n}=1 \text { in } H .
$$

The integer $n$ is called the length of the equation $(*)$, and $\sigma=e(1)+\cdots+$ $e(n)$ the exponent-sum. It is conjectured (see for example [8, Problem 2a]) that any equation $(*)$ over any group $G$, with exponent-sum $\sigma \neq 0$, has a solution over $G$. There are well-known counterexamples when $\sigma=0[6,7,9]$.

It was shown by F. Levin [5] that any positive equation $(*)$ (that is, $n=\sigma$ ) over any group $G$ has a solution over $G$, and by the second author [4] that any equation of length 3 has a solution over $G$. Equations of length 1 and 2 are well understood, so the next case to consider is that of an equation $(*)$ of length $n=4$ and exponent-sum $\sigma \neq \mp 4$.

The question of when an equation of length 4 and exponent-sum 0 has a solution is complicated, and will not be considered here. For example, if $g, h$ have distinct orders in $G$, then no solution over $G$ to the equation $g t^{2} h t^{-2}=1$ exists.

In the present paper, we show that any equation of length 4 and exponentsum 2 over any group $G$ has a solution over $G$. Since the case of exponentsum -2 follows by inverting $t$, we complete the proof of the above-mentioned

Received by the editors June 20, 1989.

1980 Mathematics Subject Classification (1985 Revision). Primary 20F05; Secondary 20F32, 20F06. 
conjecture for all equations of length less than 5 . Since we are free to alter the left-hand side of $(*)$ by conjugation in the free product $G *\langle t\rangle$, we can state our result as follows.

Theorem. Let $G$ be a group, and $a, b, c, d \in G$. Then the equation

$$
\text { atbtctd } t^{-1}=1
$$

has a solution over $G$.

If we apply the change of variable $s=t b$, the equation becomes $a s^{2} c^{\prime} s d^{\prime} s^{-1}$ $=1$, where $c^{\prime}$ denotes the element $b^{-1} c$ of $G$ and $d^{\prime}$ denotes $b^{-1} c b$, so there is no loss of generality in assuming $b=1$ in $G$. Following the method of [4], we make this assumption from now on, but retain the symbol $b$ for notational convenience.

We also note, as in [4], that there is no loss in assuming that $G$ is generated by $\{a, c, d\}$. Again we make this assumption from now on. As in [4], the method is to find enough relations among these generators to show that $G$ is abelian-by-finite (and hence residually finite). The result then follows from the famous theorem of Gerstenhaber and Rothaus.

Theorem [3,10]. Let $G$ be a locally residually finite group. Then any equation over $G$ with nonzero exponent-sum has a solution over $G$.

We prove the theorem in a series of stages, depending on which short words in the generators are relations in $G$. The first of these (Proposition 1) considers the case $c=b \quad(=1)$ in $G$, and asserts that the resulting equation $a t^{3} d t^{-1}=1$ has a solution over $G$. This result has been generalised by the first author: for any $n \geq 2$ the equation $a t^{n} d t^{-1}=1$ has a solution over $G$. This more general result will appear elsewhere [2].

\section{Preliminaries}

If the theorem is wrong, then there is a relative diagram $D$, or $\left(D, \varphi, v_{0}\right)$, in the sense of [4], representing a counterexample. This is a spherical diagram, or tesselation of the 2-sphere, $D$, with labelling function $\varphi$ on the corners of $D$, and distinguished vertex $v_{0}$. The vertices of $D$ other than $v_{0}$ are called interior vertices. The faces of $D$ are all 4-sided, with edge-orientations and corner-labels as illustrated in Figure 1. A face will be called interior if all its vertices are interior. The label of a vertex $v$ of $D$ is the list of corner labels at $v$ read in a counterclockwise direction from any starting point to make a word in $\{a, b, c, d\}$. These labels, with the unique exception of that of $v_{0}$, are relations in the group $G$.

Strictly speaking, $D$ is a spherical diagram over the 2-complex consisting of a single cell in each dimension 0, 1, 2, with the 2-cell attached as in Figure 1. The vertex-labels thus correspond to closed paths in the "labelled star graph" $\Gamma$ of this 2-complex (see Figure 2). This gives a strong restriction on the words which can arise as vertex-labels. The change-of-variable argument used in the 


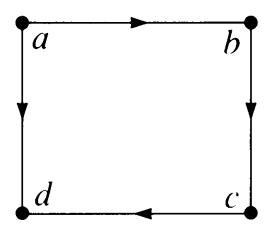

FIGURE 1

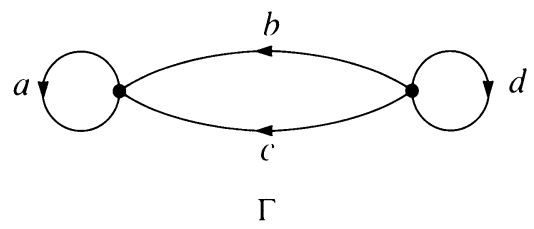

FIGURE 2

introduction to reduce to the case $b=1$ can be seen in terms of $\Gamma$ as collapse of a maximal tree.

As mentioned in the introduction, we are assuming that $G$ is generated by the elements $a, b, c, d$, and that $b=1$ in $G$. Note also that $G$ cannot be residually finite, by the Gerstenhaber-Rothaus Theorem. We shall assume this implicitly throughout the rest of the paper. Finally we make the following

Global assumption. $D$ contains the least number of faces of any relative diagram representing a counterexample.

Suppose some vertex $v$ has a label of the form $x \cdot y$, where $x$ and $y$ are nonempty words corresponding to closed paths in $\Gamma$, and we know that $x=1$ in $G$. Then it is possible to change $D$ by a diamond move as illustrated in Figure 3. (Such moves are used in special cases in [4].) Note that $y=1$ in $G$ unless $v=v_{0}$, so this move is allowable. Indeed, if $v_{0}$ is one of the vertices involved in the diamond move, then there is a unique choice for the distinguished vertex of the resulting diagram, and otherwise $v_{0}$ remains unchanged. A complete discussion of diamond moves on spherical and other diagrams is contained in [1]. In particular, it is explained there that the result of a diamond move on a spherical diagram need not in general be another spherical diagram, but may be a diagram on some other "support complex". Fortunately, this problem is avoided in our situation by the global minimality assumption stated above: if a diamond move does not give rise to a new spherical diagram, then it gives rise to at least two smaller spherical diagrams, at least one of which will be a smaller relative diagram in our situation-contrary to the global assumption.

As a particular application of diamond moves, we note that, as in [1], we have the following.

Remark 2.1. There is no vertex-label of the form $x \cdot a \cdot y \cdot a^{-1}$ for nonempty words $x, y, a$, where $x=1$ in $G$ (see Figure 4).

We next note some easy cases of the theorem. 

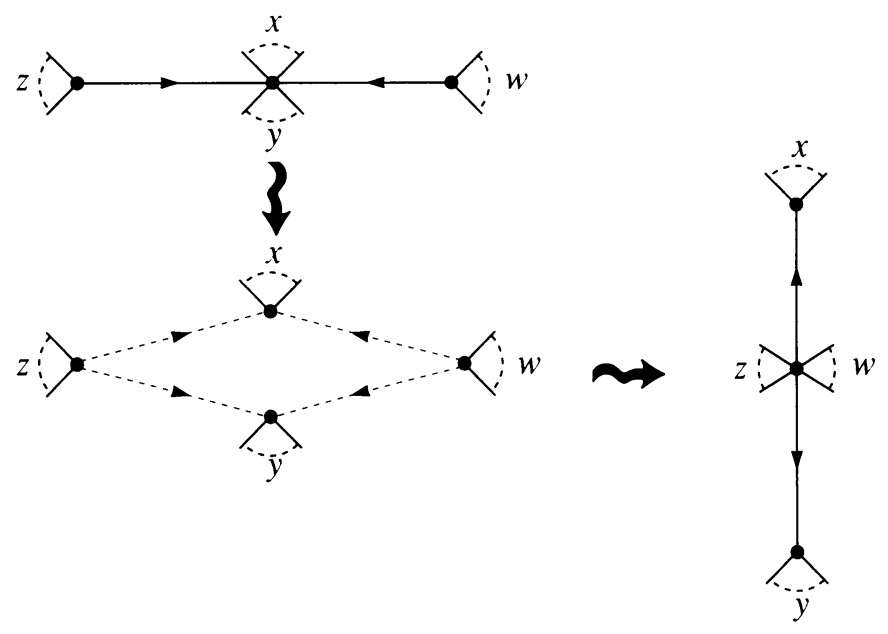

FigURe 3
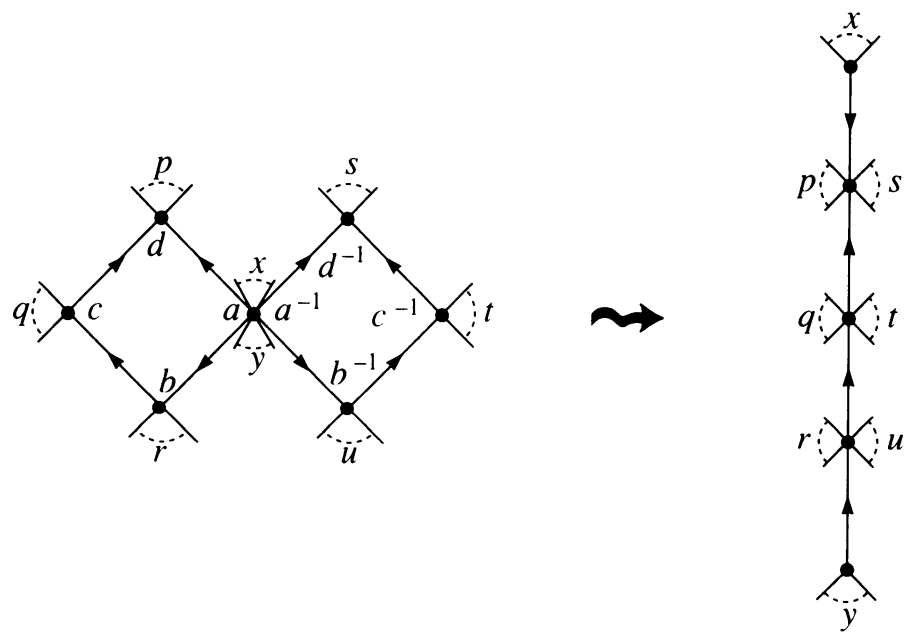

FIGURE 4

Remark 2.2. If $a=1$ or $d=1$ in $G$, then atbtctdt ${ }^{-1}$ becomes btctd or atbtc (up to conjugacy), so the equation has a solution over $G$ by Levin's Theorem.

Remark 2.3. If $c a=d b$ in $G$, then the equation has a solution in $G \mathrm{wr} \mathbb{Z}_{2}=$ $\left\langle G, s \mid s^{2}=1=[g, s h s](g, h \in G)\right\rangle$, namely $t=s a^{-1} c^{-1}$.

For

$$
\begin{aligned}
& a\left(s a^{-1} c^{-1}\right) b\left(s a^{-1} c^{-1}\right) c\left(s a^{-1} c^{-1}\right) d\left(s a^{-1} c^{-1}\right)^{-1} \\
& \quad=a a^{-1} c^{-1} c \cdot s \cdot a^{-1} c^{-1} b a^{-1} c^{-1} d c a \cdot s \\
& \quad=1 \cdot s \cdot 1 \cdot s=1 .
\end{aligned}
$$



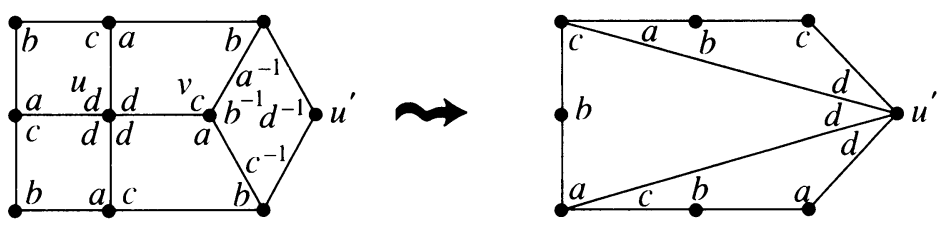

FIGURE 5

Next we note an easy fact about our relative diagrams, under the minimality assumption.

Lemma 2.4. No interior vertex of index 3 is adjacent to a source or a sink.

Proof. Suppose the interior vertex $v$ of index 3 is adjacent to a sink $u$ of index $k$ (the case of a source is similar). Then $u$ and $v$ have labels $d^{k}$, $c a b^{-1}$ respectively, up to cyclic permutation and inversion. Assuming for the moment that $u$ is interior, we have relations $c a=b$ and $d^{k}=1$ in $G$. We replace the $(k+1)$-face configuration surrounding $u$ and $v$ by a $(k-1)$-face configuration, as illustrated (in the case $k=4$ ) in Figure 5. This contradicts the assumption of minimality.

A similar argument is possible if $u$ is the distinguished vertex. Here we have $d^{k} \neq 1$ in $G$, so the label of the vertex marked $u^{\prime}$ in Figure 5 is not equal to 1 in $G$ after the adjustment. But the distinguished vertex $u$ has vanished, so we have a new diagram with distinguished vertex $u^{\prime}$.

Many of our arguments will concern diagrams obtained from the original relative diagram by some sort of amendments. These amended diagrams will have faces which are 4-, 6- or 8-gons. The principal technique is a form of curvature argument, which goes as follows. Suppose every corner of every face is given an angle (which can, in principle, be any real number). The curvature of a vertex $v$ is defined to be $2 \pi$ less the sum of angles at $v$. The curvature of a $k$-gonal face is the sum of all the angles of that face, less $(k-2) \pi$. The Euler (or Gauss-Bonnet) formula tells us that the total curvature of the diagram, summed over all the vertices and faces, is precisely $4 \pi$. We shall use only two standard methods of associating angles. One is to give each $k$-gonal corner an angle of $(k-2) \pi / k$, so that all faces have zero curvature, and we need consider only curvature of vertices. The second, dual method is to give each corner at a vertex of index $k$ an angle of $2 \pi / k$, so that vertices have zero curvature and we need only consider faces. The next result concerns this second type of curvature.

Lemma 2.5. Let $D^{\prime}$ be a tessellated 2-sphere with a distinguished vertex $v_{0}$, in which each face is a 4-gon, and no vertex other than $v_{0}$ has index less than 3. Give each corner of index $k$ an angle of $2 \pi / k$. Then there exists a positively curved face with none of its corners at $v_{0}$.

Proof. We prove this by adding up the curvature of all faces which have a corner at $v_{0}$, and noting that the result is less than $4 \pi$. If $v_{0}$ has index $k \neq 2$, then 
there are at most $k$ faces with a corner at $v_{0}$, each such face has one corner of angle $2 \pi / k$, and the other three of angle at most $2 \pi / 3$, so the curvature is at most $2 \pi / k$ per face. Summing over all such faces gives at most $2 \pi$. The same argument applies if $v_{0}$ has index 2 , unless both corners at $v_{0}$ come from the same face. But in this exceptional case (which one can easily show does not in fact arise) the unique face in question would have two corners of angle $\pi$, and two of angle at most $2 \pi / 3$, and so the curvature would be at most $4 \pi / 3$.

Hence the total curvature of all faces having a corner at $v_{0}$ is at most $2 \pi$ in all cases, and since the total curvature of all faces is precisely $4 \pi$, the result is immediate.

\section{Proof of Theorem}

Essentially, we split the proof into five cases: (i) $c=b$; (ii) $a, d$ have order 2; (iii) one of $a, d$ has order 2; (iv) $c a=b$ or $d b=c$; and (v) none of the above. We state the first four in the form of propositions.

Proposition 1. The equation $a t^{3} d=t$ has a solution over $G$.

Proof. As discussed in $\S 2$, we assume that $G$ is generated by $a$ and $d$, and that $D$ is a relative diagram representing a counterexample to the proposition, with fewest possible faces. From these assumptions we shall derive a contradiction.

Let $|a|,|d|$ denote the orders of $a, d$ respectively in $G$. By symmetry, we may clearly assume that $|a| \leq|d|$. Then we also have $|a| \geq 2$ and $|d| \geq 3$, since $G$ is not residually finite.

In addition to these assumptions, we shall impose two more. First, we assume that $D$ contains the greatest possible number of vertices of index 2 (subject to the global assumption of fewest possible faces). Second (in the case $|a|=2$ ), we assume that the number of vertices in $D$ labelled $a^{\mp 2}$ is as large as possible (subject to the previous assumptions of fewest possible faces and most possible index 2 vertices).

Our various assumptions allow us to make the following observations.

Observation 1. $D$ contains no vertex of index greater than 2 whose label involves only $b$ and $c$.

To see this, let $v$ be such a vertex. Then any vertex adjacent to $v$ has index greater than 2, since $b a^{-1}$ and $c^{-1} d$ are not labels of closed paths in $\Gamma$. Hence a diamond move as in Figure 6 will increase the number of index 2 vertices, without increasing the number of faces, contrary to assumption.

Observation 2. If $a^{2}=1$ in $G$, then no vertex label in $D$ has the form $(\text { awax })^{\mp 1}$ with $x$ a nonempty word and $w$ a word in $b$ and $c$.

Observe that $w$ is a power of $b^{-1} c$ (positive or negative). A sequence of diamond moves in as Figure 7 increases the number of vertices of $D$ labelled $a^{\mp 2}$ without decreasing the total number of index 2 vertices, nor increasing the number of faces of $D$. This contradicts our assumptions. 

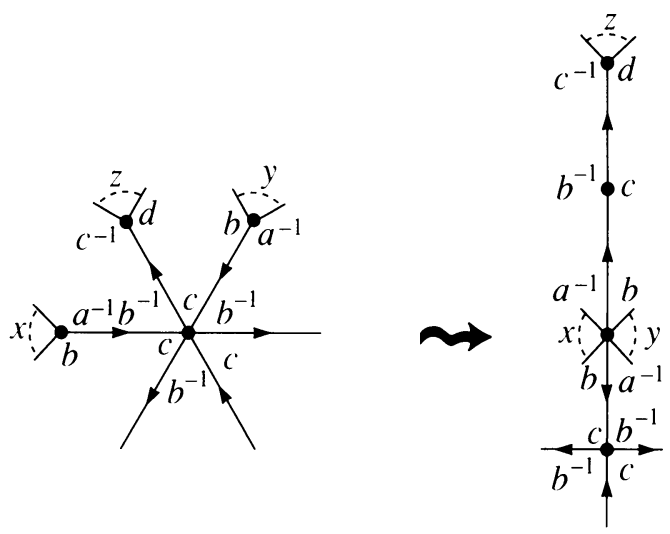

FIGURE 6
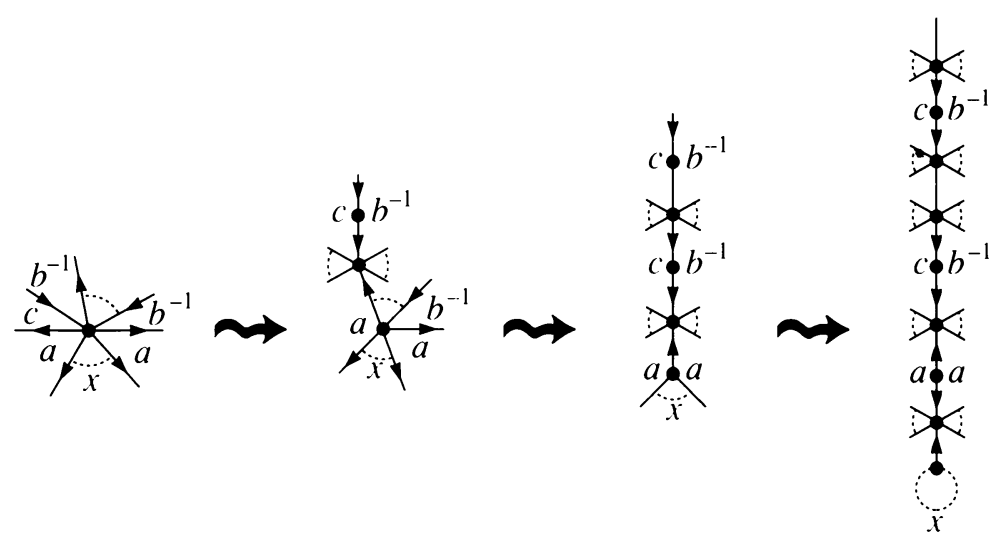

FIGURE 7

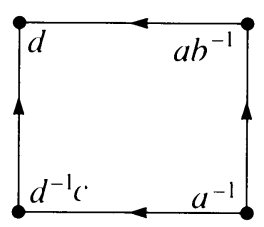

FIGURE 8

Form an amended diagram $D^{\prime}$ by removing from $D$ all interior vertices of index 2, together with any incident edges. Since two vertices of index 2 can share a common incident face only if they are the $a$ - and $c$-corners of that face, we see that the faces of $D^{\prime}$ are 4-gons, with corner labellings and edge orientations either as in $D$ or as illustrated in Figure 8 (up to orientation), or in the case $a^{2}=1$ as illustrated in Figure 9 (up to orientation). In particular, the corner-labels are all subwords of $\left(a b^{-1} d^{-1} c\right)^{\mp 1}$, and if $a^{2} \neq 1$ in $G$ then the only corner-labels of length greater than 1 are $\left(a b^{-1}\right)^{\mp 1}$ or $\left(d^{-1} c\right)^{\mp 1}$. 


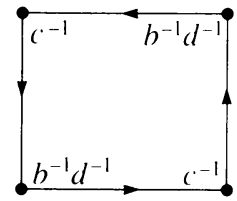

(i)

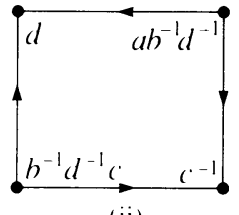

(ii)

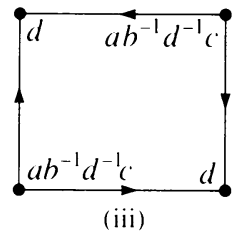

(iii)

FIGURE 9
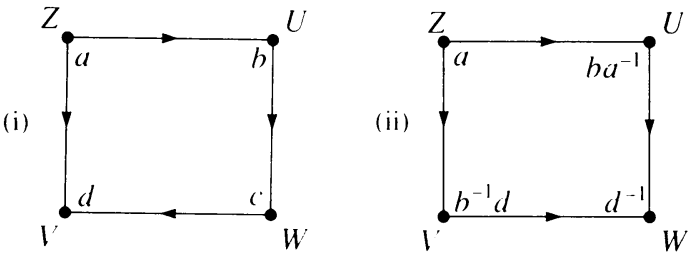

FIGURE 10

Next, note that $D^{\prime}$ has no interior vertex of index less than 3 . This is clear if $a^{2} \neq 1$, for then the label of such a vertex would yield a relator of length at most 2 between $a$ and $d$, and $G$ would be cyclic. If $a^{2}=1$, then any such label would yield a relation making $G$ cyclic, with the single exception of $\left(a b^{-1} d^{-1} c\right)^{\mp 2}$, which would make $G$ dihedral. In all cases, $G$ is residually finite, a contradication.

Thus, if we give each corner in $D^{\prime}$ an angle of $2 \pi / n$, where $n$ is the index of the vertex in $D^{\prime}$, there must be a positively curved interior face, $P$ say, by Lemma 2.5. At this point it is convenient to split the argument into two cases, depending on $|a|$.

Case 1. $|a| \geq 3$. At lest one vertex of $P$ has index 3 , and (after deletion of symbols $b, c)$ its label is a cyclically reduced word of length 3 in $\{a, d\}$. Since $G$ is not cyclic, the only possible labels are $a^{\mp 3}$ and $d^{\mp 3}$. Without loss of generality we assume the label is $a^{3}$. Thus $P$ is as in Figure 10 ((i) or (ii)), with vertex $Z$ of index 3 .

Suppose first that $P$ is given by Figure 10(i). Then no vertex of $P$ other than $Z$ can be a source or a sink, so $U, V, W$ have index at least 4 . But at least two of them have index 4 , with the third of index 4 or 5 , since $P$ is positively curved.

Suppose $U$ has index 4. The label of $U$ begins $d^{ \pm 1} \cdot b$ or $\left(c^{-1} d\right) \cdot b$, and (since $d^{2} \neq 1$ ) the only possibility which does not force $G$ to be abelian-by-finite is $d \cdot b \cdot\left(c^{-1} d\right) \cdot d$. A similar analysis applies if $V$ has index 4 , so in any case we can deduce that $d^{3}=1$. If $U$ and $W$ have index 4 , then the label of $W$ begins $d^{-1} \cdot c$, and the only possibilities force $G$ cyclic, a contradiction. If $U$ and $V$ have index 4 , then $W$ must have index 5 , and a label beginning $d^{-1} \cdot c \cdot a$ or $d^{-1} \cdot c \cdot\left(a b^{-1}\right)$. In all cases, $G$ is either finite or cyclic, a contradiction.

Hence we may assume $V$ and $W$ have index 4 , while $U$ has index 5 . The only possibility for the label of $W$ is $c \cdot a \cdot a \cdot\left(a b^{-1}\right)$, so $U$ has a label beginning $d \cdot b \cdot a^{-1}$ or $\left(c^{-1} d\right) \cdot b \cdot a^{-1}$. In all cases $G$ is cyclic, a contradiction.

Hence $P$ must be as in Figure 10(ii). Again $U$ and $V$ must have index at least 4 , but this time we must consider the possibility that $W$ has index 3 , 
and label $d^{-3}$. At least one of $U, V$ has index less than 6 , and by symmetry we may assume it is $U$. The label of $U$ has a symbol $\left(b a^{-1}\right)$ preceded by either $d$ or $\left(c^{-1} d\right)$ and followed by either $c^{-1}$ or $\left(c^{-1} d\right)$. In all cases, $G$ is abelian-by-finite, a contradiction.

Hence $W$ has index greater than 3 . Next suppose that $U$ has index 4 . Since $U$ has label beginning $d \cdot\left(b a^{-1}\right)$ or $\left(c^{-1} d\right) \cdot\left(b a^{-1}\right)$ and $G$ is not abelian-byfinite, we can eliminate all labels for $U$ except $\left(\left(c^{-1} d\right) \cdot\left(b a^{-1}\right)\right)^{2}$. But then $W$ has index 4 or 5 , with label beginning $d^{-2}$. In all cases $|d| \leq 5$, and since $a^{3}=\left(d a^{-1}\right)^{2}=1$ it follows that $G$ is finite, a contradiction.

A similar analysis applies if $V$ has index 4 , so in all cases we have obtained a contradiction, completing the proof in Case 1 .

Case 2. $|a|=2$. We first list the possible labels of interior vertices of index 3 in $D^{\prime}$.

Observation 3. (i) If $d^{3} \neq 1$, then any interior vertex of $D^{\prime}$ of index 3 has label $\left(c^{-1} d b a^{-1}\right)^{3}$.

(ii) If $d^{3}=1$ then any interior vertex of $D^{\prime}$ of index 3 has label $d^{\mp 3}$, $\left(d \cdot(d b) \cdot\left(c^{-1} d\right)\right)^{\mp 1},\left(c^{-1} d b\right)^{\mp 3}$, or $\left(\left(c^{-1} d b\right)\left(c^{-1} d\right)(d b)\right)^{\mp 1}$.

(iii) No two interior vertices of index 3 in $D^{\prime}$ are adjacent.

To see (i), note that the relation obtained from such a vertex label by deleting symbols $b$ and $c$ contains at most three occurrences each of $a$ and $d$. We can deduce that $G$ is abelian-by-finite unless the relation is $a^{2}$ or $(a d)^{3}$. But $a^{2}$ is ruled out by Observation 2 , and the stated label is the only way that the relation $(a d)^{3}$ can arise.

For (ii), a similar analysis shows that the relator must by $d^{3}$, which can arise only from the labels listed. Statement (iii) follows immediately from (i) and (ii).

Suppose first that $d^{3} \neq 1$. Then by Observation 3(i) $P$ must be as in Figure 11 , with $Z$ of index 3 . Suppose that the index of $U$ is no greater than that of $V$ (the argument is similar in the other case). Either $W$ has index 3, and $U$ has index at most 5 , with label beginning $d^{-3}$, or $U$ has index 4 , with label beginning $d^{-2}$. In either case the label contains at most five occurrences of $d$, and at most two of $a$. From any relation of this form, together with $a^{2}=(a d)^{3}=1$, we can deduce that $G$ is finite, a contradiction.

Hence $d^{3}=1$. From this, we deduce that any interior vertex of $D^{\prime}$ whose label involves an a must have index at least 6 . To see this, note first that

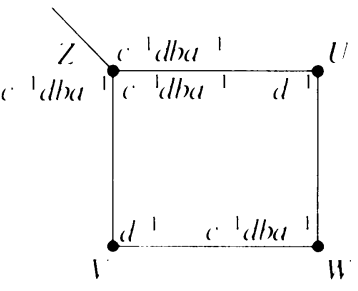

FIGURE 11 


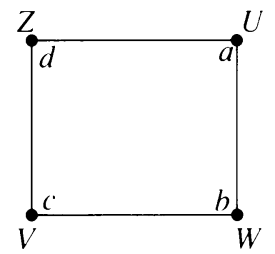

(i)

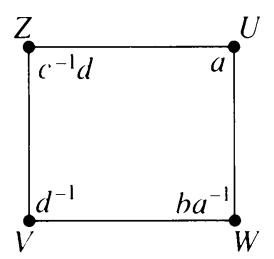

(iv)

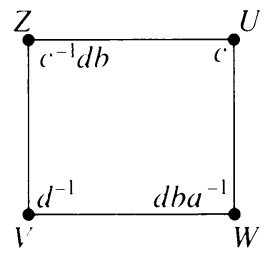

(ii)

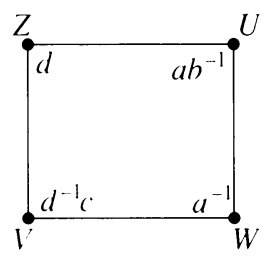

(v)

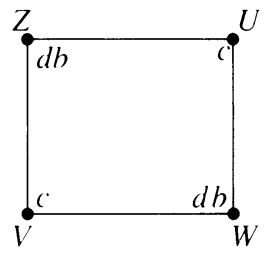

(iii)

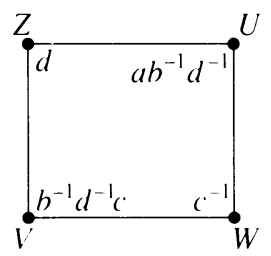

(vi)

FIGURE 12

the label also involves $d$, by Observation 2. If the index is less than 6 , then, by Observation 2 and the relations $a^{2}=1=d^{3}$, the derived relation can be rewritten as a product of between one and five words of the form $a d^{\mp 1}$. But any such additional relation implies that $G$ is abelian-by-finite, contrary to hypothesis.

By Observation 3(ii), the face $P$ is as in Figure 12, with $Z$ a vertex of index 3. In Figure 12(i) and (vi) the vertex $U$ has index at least 6 , while neither $V$ nor $W$ has index 3 by Observations 3(ii) and (iii). In Figure 12(ii), (iv), and (v) the vertex $W$ has index at least 6 , while neither $U$ nor $V$ can have index 3 by Observation 3(iii). In Figure 12(iii) the vertex $Z$ has label $(d b) \cdot\left(c^{-1} d\right) \cdot d$ or $(d b) \cdot\left(c^{-1} d b\right) \cdot\left(c^{-1} d\right)$. In either case the vertex $V$ has label involving $a$, so index at least 6 , while $U$ cannot have index 3 by Observation 3(iii). Moreover if $W$ has index 3, then the same argument shows that $U$ has index at least 6. In all cases, we have contradicted the assertion that $P$ is positively curved. This completes the proof.

Proposition 2. Suppose $a$ and $d$ are elements of order 2 in $G$. Then the equation atbtctd $=t$ has a solution over $G$.

Proof. By Proposition 1 we have $c \neq 1$ in $G$.

Note first that in this case no interior vertex of $D$ has index 3 , for then we would have a relation in $G$ of the form $a^{\mp 3}$ or $d^{\mp 3}$ (contradicting $a \neq 1 \neq d$ ) or $a^{\mp 1}\left(b^{-1} c\right)^{\mp 1}$ or $d^{\mp 1}\left(b c^{-1}\right)^{\mp 1}$ (which would imply that $G$ is dihedral).

Note also that, since $b=1 \neq c$, any interior vertex of index 2 in $D$ is a sink or a source. In particular, no two such vertices are adjacent. Form an amended diagram $D^{\prime}$ by removing all interior vertices of index 2 in $D$, together with any incident edges. Then the faces of $D^{\prime}$ are 4-gons, with corner-labels and edge-orientations either as in $D$ or as illustrated in Figure 13 (modulo change of orientation), where $e=c a$ and $f=d b$. 

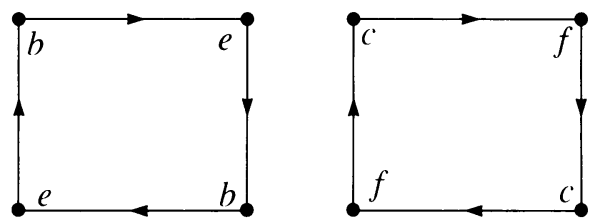

FIGURE 13
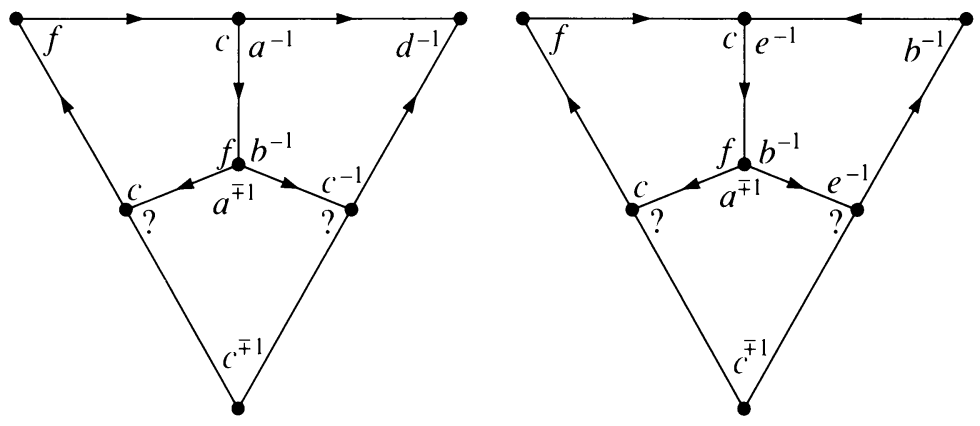

FIGURE 14

If $D^{\prime}$ has an interior vertex $v$ of index 2 , then $v$ has index 4 in $D$, and label $\left(a^{-1} c^{-1} d b\right)^{\mp 1}$. But then the equation has a solution over $G$ by Remark 2.3. Hence no interior vertex of $D^{\prime}$ has index less than 3 .

Next consider a vertex $v$ of index 3 in $D^{\prime}$. The only possible labels (up to cyclic permutation and inversion) for $v$ are:

(i) $a b^{-1} e, d f c^{-1}$;

(ii) $a f^{-1} e, d f e^{-1}$;

(iii) $f a^{\mp 1} c^{-1}, e b^{-1} d^{\mp 1}$;

(iv) $f a^{\mp 1} b^{-1}, e c^{-1} d^{\mp 1}$.

Either label of (i) as a relation in $G$ would contradict $b \neq c$; while either label of (ii) would imply that $a=c$ or $d=c$, and hence that $G$ is dihedral, a contradiction. Either label of (iii) would imply $c a=d b$, so that the equation would have a solution over $G$, a contradiction. If both labels of (iv) are relations in $G$, then $G$ is residually finite, and the equation has a solution over $G$. Hence only one label of (iv) can occur, and by symmetry we may assume that only the first occurs. In other words, every interior vertex of $D^{\prime}$ of index less than 4 has index 3 and label $f a^{\mp 1} b^{-1}$.

Give each corner at each vertex of index $k$ an angle of $2 \pi / k$. Then by Lemma 2.5 there is an interior face $F$ in $D^{\prime}$ with positive curvature. Note that every vertex of $D^{\prime}$ adjacent to one labelled $f a^{\mp 1} b^{-1}$ has label involving the letter $c$ or $e$ (Figure 14), so cannot be interior of index 3. Hence either one or two vertices of $F$ have index 3, and adjacent to such a vertex is a vertex of $F$ of index 4 or 5 containing $c$ or $e$ in its label $l$. Now $\{a, b, d, f\}$ generate a cyclic subgroup of order 2 in $G$, so it follows that, if the number of occurrences of $c$ and $e$ in $l$ is one or two, then $G$ is abelian or dihedral, a contradiction. 
On the other hand, no cyclically reduced vertex label in $D$ can give rise to a label in $D^{\prime}$ containing a subword $\left(c^{-1} e\right)^{\mp 1}$, so $c$ and $e$ cannot occur four times in $l$, and indeed the only possibilities for $l$ are $\left(c e^{-1}\right)^{\mp 1} d^{\mp 1} x y^{-1}$, where $x \in\{c, e\}$ and $y \in\{b, f\}$. But any one of these four possibilities implies that $G$ is either cyclic or dihedral, a contradiction. This completes the proof.

Proposition 3. If $a^{2}=1$ or $d^{2}=1$ in $G$, then the equation atbtctd $=t$ has $a$ solution over $G$.

Proof. Without loss of generality, we may assume that $a^{2}=1$. By Propositions 1 and 2 we have $c \neq 1$ and $d^{2} \neq 1$ in $G$.

As in the proof of Proposition 1, we assume in this case that $D$ has the greatest possible number of interior vertices of index 2 (subject to the global assumption that $D$ has fewest possible faces). Any such vertex has label $a^{\mp 2}$.

This latter assumption implies that $a^{\mp 2}$ never appears as a proper subword of any vertex-label, for otherwise a diamond move could create an extra vertex of index 2 (see Figure 15). Hence also any subword $(d b)^{\mp 1}$ of a vertex-label arises only when an adjacent vertex has index 2 .

Form an amended diagram $D^{\prime}$ from $D$ by removing all interior vertices of index 2 , together with any incident edges. The faces of $D^{\prime}$ are 4-gons, with edge-orientations and corner-labellings either as in $D$ or as shown in Figure 16 (up to orientation), where $f=d b$.

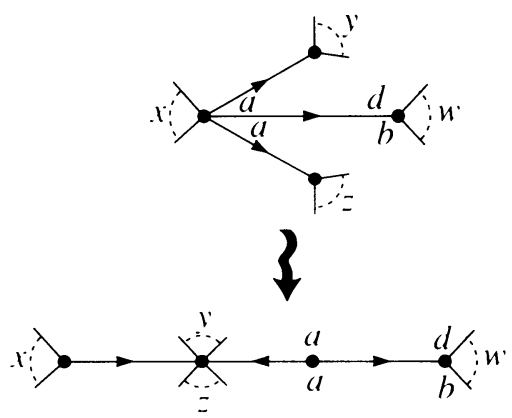

FIGURE 15

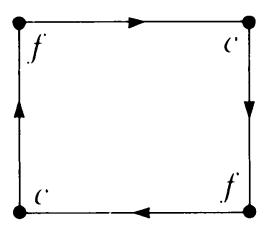

FIGURE 16

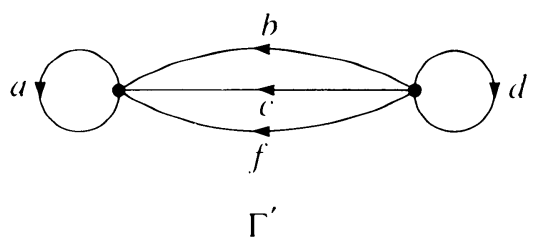

FIGURE 17 
The vertex labels of $D^{\prime}$ thus correspond to closed paths in the "amended star graph" $\Gamma^{\prime}$ indicated in Figure 17.

Now any interior vertex of index 2 in $D^{\prime}$ must arise from a vertex of index 3 in $D$ which is adjacent to a source, contradicting Lemma 2.4. Hence all interior vertices in $D^{\prime}$ have index at least 3. Moreover, vertices of index 3 must exist, by Euler's formula.

Now consider an interior vertex of $D^{\prime}$ of index 3. Possible labels for such a vertex (up to cyclic permutation and inversion) are: $a^{3}, d^{3}, a^{\mp 1} b^{-1} c, a^{\mp 1} b^{-1} f$, $a^{\mp 1} c^{-1} f, d^{\mp 1} b c^{-1}$, or $d f c^{-1}$. Since the label is equal to 1 in $G$, we can eliminate some of these possibilities immediately. For example, if $a^{3}=1$ then $a=1$, contradicting Remark 2.2; if $a^{\mp 1} b^{-1} f=1$ then $d^{2}=1$, a contradiction; while if $a^{\mp 1} c^{-1} f=1$ then $c a=d b$, and the result follows from Remark 2.3. We group the remaining possible labels into three classes:

$$
A=\left\{d^{3}\right\} ; \quad B=\left\{a^{\mp 1} b^{-1} c\right\} ; \quad C=\left\{d^{\mp 1} b c^{-1}, d f c^{-1}\right\} .
$$

Note that $G$ is cyclic if labels from both $B$ and $C$ occur, so we may assume this does not happen.

Suppose first that some interior vertex of $D^{\prime}$ has a label from $C$. Then we may assume that no interior vertex has a label containing the letter $a$ precisely once (for otherwise $G$ is cyclic). Since labels are closed paths in $\Gamma^{\prime}$ not containing $a^{\mp 2}$ as a cyclic subpath, any vertex whose label involves the letter $a$ must have index at least $3 p \geq 6$ (where $p$ denotes the number of occurrences of $a$ ), and $p \geq 2$ if the vertex is interior.

Let $v$ be a vertex of index 3 in $D^{\prime}$. A vertex $u$ is associated to $v$ if $u$ is adjacent to $v$ and some face of $D$ has its $a$-corner at $u$ and its $d$-corner at $v$. Note that at least one vertex (not necessarily interior) is associated to $v$. Moreover, a vertex $u$ which is associated to $p \geq 1$ vertices of index 3 contains the letter $a$ at least $p$ times in its label, so has index at least

$$
\max \{6,3 p\} \geq 4+p,
$$

unless it is the distinguished vertex, when it has index at least $3 p \geq p+2$. Let $V$ be the number of vertices of $D^{\prime}$, and $W$ the number of interior vertices of index 3 . We thus have a minimum of $(V-W-1)$ vertices of index 4 or more, and the distinguished vertex has index at least 1 . Moreover, for each vertex $v$ of index 3 we can increase this lower bound by at least 1 for the vertex $u$ associated to $v$. Thus the sum of all the indices is at least $3 W+4(V-W-1)+W+1=4 V-3$, which contradicts Euler's formula.

Hence we may assume that no interior vertex has a label in $C$. Suppose next that some interior vertex has a label in $B$, so that $c=a$ in $G$. We proceed more or less as before.

If $v$ is a vertex of index 3 in $D^{\prime}$, then a vertex $u$ is associated to $v$ if $u$ is adjacent to $v$ and some face of $D$ has $u$ and $v$ as its $a$-and $d$-corners (not necessarily in that order). Thus a vertex in $A$ has three associated vertices, while a vertex in $B$ has one associated vertex. 

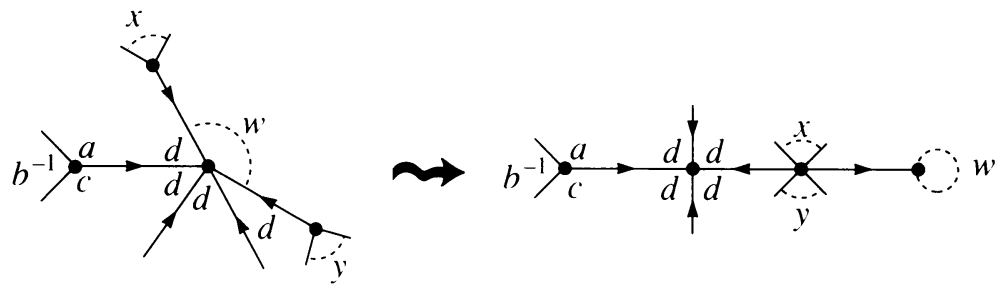

FIGURE 18

Suppose that $u$ is a vertex of $D^{\prime}$ which is associated to $s$ vertices in class $A$ and to $t$ vertices in class $B$, where $s+t>0$. To each $A$ - (resp. $B$-) vertex to which $u$ is associated, there corresponds a cyclic subword $(c a)^{\mp 1}\left(\right.$ resp. $\left.d^{\mp 1}\right)$ of the label of $u$. Since the letter $a$ cannot be followed in a label by $c, d^{\mp 1}$, or $a^{-1}$, it follows that $u$ has index at least $3 s+t$ in $D^{\prime}$. If the index of each such interior $u$ is at least $4+s+t$, then we can argue as before to obtain a contradiction. Hence some such interior vertex $u$ has index less than $4+s+t$.

Suppose first that $t=0$. Then $0<3 s<4+s$, so $s=1$ and $u$ has index precisely 4 in $D^{\prime}$. The label of $u$ corresponds to a closed path in $\Gamma^{\prime}$ of length 4 , beginning $c a$. The possibilities are

$$
c a b^{-1} d^{\mp 1}, \quad c a c^{-1} d^{\mp 1}, \quad c a f^{-1} d^{-1} .
$$

If any one of these words is equal to 1 in $G$, then it follows from the relations $a^{2}=1=b=c a$ that $d^{2}=1$ in $G$, a contradiction.

Hence $t>0$. Note that $u$ cannot be a sink by Lemma 2.4, but the label of $u$ is a word of length at most $t+s+3$, and at least $3 s+t$, containing at least $t$ letters $d^{\mp 1}$ and at least $s$ subwords $(c a)^{\mp 1}$. The only possibilities are that $s=0$ or $s=1$, and that the label of $u$ (in $D$ ) has the form $d^{k} w$, up to cyclic permutation and inversion, for some integer $k \geq t$ and some nonempty word $w$ in $\{a, b, c\}$. Since $\{a, b, c\}$ generate a cyclic subgroup of order 2 in $G$, and $G$ is not cyclic, it follows that $w=1$ in $G$. But then we can alter $D$ by a diamond move (Figure 18) to place a sink adjacent to a vertex of index 3, contradicting Lemma 2.4 .

In either case we have a contradiction, so the only interior vertices of index 3 in $D^{\prime}$ are sinks. Since at least one such vertex exists, we deduce that $d^{3}=1$ in $G$.

Now form yet another amended diagram $D^{\prime \prime}$ by removing all interior vertices of index 3 from $D^{\prime}$, together with any incident edges. Then the faces of $D^{\prime \prime}$ are 4-gons as in $D^{\prime}$, and 6-gons with edge-orientations and corner-labellings as shown in Figure 19 (up to orientation), where $e=c a$. Vertex labels are closed paths in the star graph $\Gamma^{\prime \prime}$ shown in Figure 20.

Now $D^{\prime \prime}$ has no interior vertex of index 2 , since such a vertex could arise only from an interior vertex of index 3 in $D^{\prime}$ other than a sink.

Give each corner of $D^{\prime \prime}$ an angle of $\pi / 2$ or $2 \pi / 3$, according to whether it belongs to a 4-gonal or 6-gonal face of $D^{\prime \prime}$. By Euler's formula there exists a positively curved interior vertex, and the label of such a vertex is a closed path 


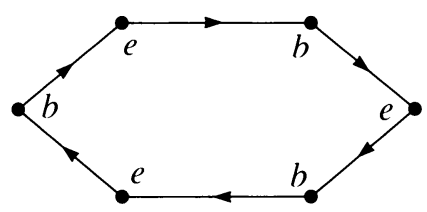

FIGURE 19

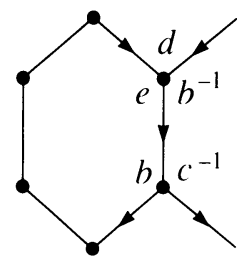

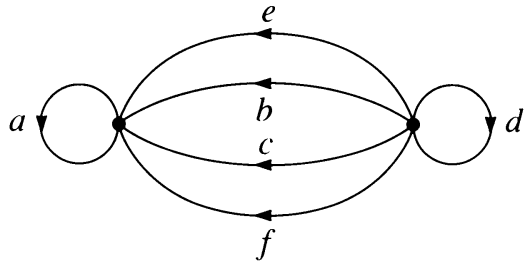

FIGURE 20

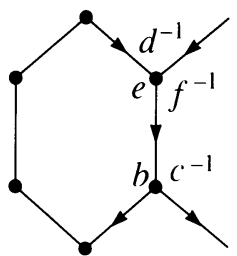

FIGURE 21

of length 3 in $\Gamma^{\prime \prime}$ containing the letter $e$ precisely once (if $e$ does not appear in the label, then the vertex already had index 3 in $D^{\prime}$, so would have been removed in forming $D^{\prime \prime}$ ). Up to cyclic permutation and inversion, the possible labels are:

$$
e c^{-1} d^{\mp 1}, \quad e b^{-1} d^{\mp 1}, \quad e f^{-1} d^{\mp 1} .
$$

Of these, $e f^{-1} d$ does not correspond to a reduced word in $\{a, b, c, d\}$, so can be eliminated. We can also eliminate $e b^{-1} d^{-1}$ by Remark 2.3 , and $e c^{-1} d^{\mp 1}$ by means of the relations $b=1=a^{2}=d^{3}$. Hence every positively curved interior vertex of $D^{\prime \prime}$ has angle-sum at least $5 \pi / 3$ and label $e b^{-1} d$ or $e f^{-1} d^{-1}$ and we deduce that $c=d^{-1} b a^{-1}=d^{-1} a$ in $G$.

Now any positively curved interior vertex $v$ in $D^{\prime \prime}$ has two edges coming in and one edge going out. We say that the terminal vertex $u$ of the out-edge is associated to $v$. If $u$ is associated to $p \geq 1$ distinct, interior vertices of angle-sum less than $2 \pi$, then the label of $u$ contains at least $p$ distinct cyclic subwords of the form $\left(c^{-1} b\right)^{\mp 1}$ or $\left(e^{-1} b\right)^{\mp 1}$, with the $b$-corners belonging to 6-gonal faces (Figure 21). It follows that $u$ has index at least $2 p$ and anglesum at least $7 p \pi / 6$. In particular, if $p \geq 3$ then the angle-sum is at least $2 \pi+p \pi / 2$. By the Euler formula again, there must be at least three vertices $u$ with the following property: $u$ is associated to $p \leq 2$ positively curved interior vertices, and the angle-sum at $u$ is less than $2 \pi+p \pi / 3$. At least one such $u$ is interior, so its label is a relation in $G$.

If $p=2$, then the only possibilities for the label are $\left(c^{-1} b\right)^{ \pm 2}$ and $c^{-1} b e^{-1} b$, and it follows that $G$ is finite or cyclic. If $p=1$ then the label cannot contain the letter $e$, for then it would have to have length 3 , and the only possibility is $e a b^{-1}$, contradicting $b \neq c$. Possible labels are $c^{-1} b a^{\mp 1}, c^{-1} b a^{\mp 2},\left(c^{-1} b\right)^{2}$, $c^{-1} b c^{-1} f$, and $c^{-1} b f^{-1} b$. Of these, the last cannot occur, since it does not correspond to a reduced word in $\{a, b, c, d\}$; either of the first two as relations in $G$ imply that $d=1$, while either of the third and fourth imply that $G$ is finite. 
In all cases we have obtained a contradiction, and the result follows.

Proposition 4. Suppose that either $b=c a$ or $c=d b$ in $G$. Then the equation atbtctd $t^{-1}=1$ has a solution over $G$.

Proof. If both equations hold, then $G$ is cyclic, so we may assume that only one of them holds. By symmetry, it suffices to consider the case $b=c a \quad(=1)$. Since $G$ is not cyclic, we also have $b \neq d c$. By Proposition 3 we can assume $a^{2} \neq 1$, so also $c \neq b a$. Thus the only possible labels for interior vertices of index 3 in $D$ are $a^{3}, d^{3}, c a b^{-1}$ (up to cyclic permutation and inversion). Moreover, by Propositions 1-3 we may assume that none of $a^{2}, d^{2}, c b^{-1}$ vanish in $G$, so no interior vertex of $D$ has index 2 .

Subject to the existing assumption that $D$ has as few faces as possible, we impose the additional assumption that it contains the maximum possible number of vertices labelled $c a b^{-1}$.

Consider the subgraph $\Lambda$ of $D^{(1)}$ consisting of all vertices labelled $c a b^{-1}$ and all edges joining them. We note the following properties of $\Lambda$.

(1) If two vertices of $\Lambda$ lie on opposite corners of a face $F$ of $D$, then a third corner of $D$ is also a vertex of $\Lambda$.

Proof. Clearly only the $a$ - and $c$-corners of $F$ are involved. But then the $b$ corner of $F$ has a subword $\left(a b^{-1} c\right)^{\mp 1}$ in its label. If this is not already a vertex of $\Lambda$, then it can be made into one by a diamond move (see Figure 22). This increases the number of such vertices by at least 1 , contrary to the assumption of maximality.

(2) Every vertex of $\Lambda$ has index at most 2 in $\Lambda$.

Proof. Every vertex of $\Lambda$ has index 3 in $D$, and one of the adjacent vertices has the letter $d$ in its label, so cannot belong to $\Lambda$.

(3) $\Lambda$ is a forest.

Proof. Otherwise, by (2), some component of $\Lambda$ is a cycle, of length $k$ say. Let $v_{1}, \ldots, v_{k}$ denote the vertices of this cycle, in cyclic order. Each $v_{i}$ is adjacent
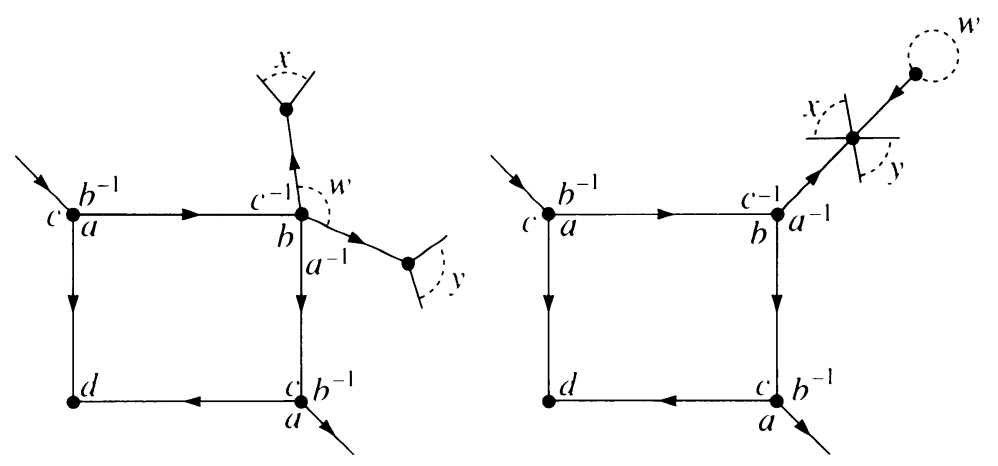

FIGURE 22 
to a unique vertex $u_{i}$ not in $\Lambda$. Moreover $u_{i+2}=u_{i}$ for all $i(\bmod k)$. Indeed, there is a face $F_{i}$ whose $a-, b$-, $c$-, and $d$-corners are $v_{i}, v_{i+1}, v_{i+2}$, and $u_{i}$ respectively (see Figure 23). Hence $k$ is even, and $u_{2}=\cdots=u_{k}$ is a sink, contradicting Lemma 2.4 .

By (1), (2), and (3) we see that $\Lambda$ is a disjoint union of chains, no two of whose endpoints have a common incident face in $D$. We can thus form an amended diagram $D^{\prime}$ by removing from $D$ all vertices of $\Lambda$ and all incident edges. The faces of $D^{\prime}$ are either 4-gons as in $D$, or 6-gons labelled as in Figure 24 (up to orientation).

Now no interior vertex of $D^{\prime}$ can have index 2 , for otherwise it would have been a vertex of $\Lambda$, or a sink in $D$ adjacent to a vertex of $\Lambda$, or we can deduce a relation $a^{2}=1$ or $d^{2}=1$ in $G$.

Any interior vertex of index 3 in $D^{\prime}$ is either a sink or a source, for otherwise it has a label (up to cyclic permutation and inversion) $\left(a b^{-1}\right)\left(d^{n}\right) c$, $\left(a b^{-1}\right)\left(d^{n}\right) b,\left(a b^{-1}\right) c a,\left(a b^{-1}\right) c\left(b^{-1} c\right)$, or $\left(b^{1} c\right) b^{-1} c$. In the first case we can increase the number of vertices of $\Lambda$ by a diamond move as in Figure 22, contrary to our maximality assumption. In the other cases we can deduce that $G$ is cyclic, again a contradiction.

We give angles of $\pi / 2$ or $2 \pi / 3$ to the corners of 4-gons and 6-gons of $D^{\prime}$ respectively. Then any positively curved interior vertex of $D^{\prime}$ has label one of

$$
a^{3}, \quad a^{2}\left(c^{-1} b\right), \quad a\left(c^{-1} b\right)^{2}, \quad\left(d^{k}\right)\left(d^{l}\right)\left(d^{m}\right) .
$$

Moreover, the last of these is possible only if $k=l=m=\mp 1$, for otherwise the vertex is adjacent in $D$ to a vertex of $\Lambda$, or its label in $D$ is nonreduced. Hence at least one of $a, d$ has order 3 in $G$.

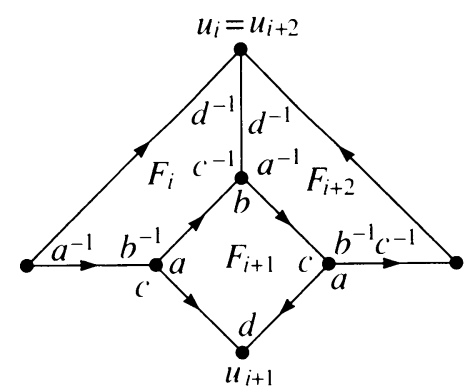

FIGURE 23

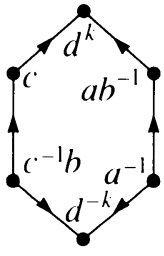

(i)

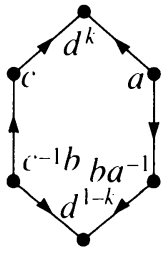

(ii)

FIGURE 24 


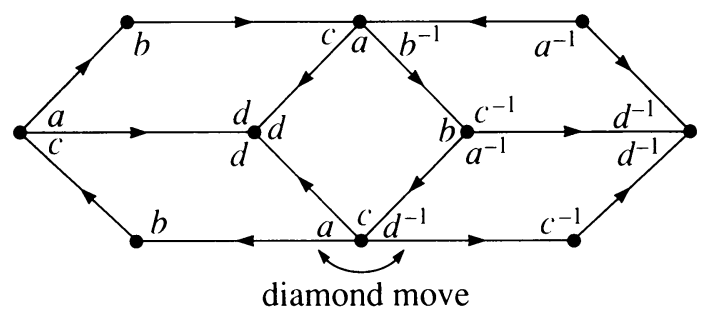

FIGURE 25

Note also that any interior sink of index 3 in $D^{\prime}$ is surrounded entirely by 4-gons, for otherwise we have a configuration in $D$ as in Figure 25, and a diamond move along the arrowed edges creates a vertex labelled $c a b^{-1}$ adjacent to a sink, contrary to Lemma 2.4. Another consequence of this is that no such sink is adjacent to a source, so no two interior vertices of index 3 in $D^{\prime}$ are adjacent.

Let $v$ be a positively curved interior vertex of $D^{\prime}$. Then the angle-sum of $v$ is $2 \pi-p \pi / 6$, where $p=1,2$, or 3 is the number of 4-gonal corners at $v$. We say that a vertex $u$ is associated to $v$ if $u$ is joined to $v$ by the $(a d)$ edge of some 4-gon in $D^{\prime}$. It may be necessary to count such vertices with multiplicity, so if $u$ is joined to $v$ by two or three such edges, we regard $u$ as being associated to $v$ twice or three times.

Now suppose $u$ is a vertex associated to $q>0$ (not necessarily distinct) interior vertices of angle-sum less than $2 \pi$. Then the label of $u$ contains $q$ distinct cyclic subwords of the form $(d b)^{\mp 1},(d c)^{\mp 1},\left[d\left(b a^{-1}\right)\right]^{\mp 1}$, or $(c a)^{\mp 1}$. It readily follows that $u$ has index at least $\min \{2 q, 4\}$ in $D^{\prime}$ and hence anglesum at least $\min \{q \pi, 2 \pi\}$. Since the total curvature of $D^{\prime}$ is $\pi$, we can find an interior vertex $u$, associated to $q \geq 0$ interior vertices of angle-sum less than $2 \pi$, such that the angle-sum of $u$ is strictly less than $2 \pi+q \pi / 6$.

Thus $q \pi<2 \pi+q \pi / 6$, so $q \leq 2$. If $q=1$ then $u$ has index 4 , with all incident faces being 4-gons. The label of $u$ is thus a cyclically reduced word of length 4 in $\{a, b, c, d\}$ beginning $d b$ or $c a$. But any such word, imposed as a relation in $G$ together with $b=1=c a$, implies that $G$ is cyclic, a contradiction.

Hence $q=2$, and the label of $u$ is a cyclically reduced word of length 4 in $\left\{a, b, c, d,\left(b a^{-1}\right),\left(b^{-1} c\right)\right\}$, beginning $d b, d c, d\left(b a^{-1}\right)$, or $c a$, and containing at most one occurrence of $\left(b a^{-1}\right)$ or $\left(b^{-1} c\right)$. Since $b=c a$ in $G$ we may replace any occurrence of $\left(b a^{-1}\right)$ by $c$ or $\left(b^{-1} c\right)$ by $a^{-1}$ to get a word $w$ of length 4 in $\{a, b, c, d\}$. Now $w$ need not be (cyclically) reduced, but since at most one letter of $w$ arose by substitution, at most one pair of letters in $w$ can cancel, and $w$ is not freely equal to the empty word. Arguing as before, we obtain that $G$ is cyclic, a contradiction. This completes the proof.

Theorem. Let $G$ be a group, and $a, b, c, d$ elements of $G$. Then the equation

$$
\text { atbtctd } t^{-1}=1
$$

has a solution over $G$. 


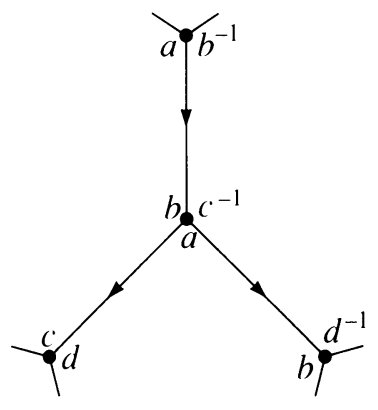

FIGURE 26

Proof. As usual, assume $b=1$ and $D$ is a relative diagram for a counterexample with fewest possible faces. By Propositions $1-4$ we have $d b \neq c \neq b \neq c a$ and $a^{2} \neq 1 \neq d^{2}$ in $G$. Thus the only possible labels for interior vertices of $D$ of index less than 4 are (up to cyclic permutation and inversion): $a^{3}, d^{3}, b a c^{-1}$, and $d c b^{-1}$. If both $b a=c$ and $d c=b$ in $G$, then $G$ is cyclic, a contradiction so at most one of them holds. When one does we can assume without any loss that it is $b a=c$.

Observe that no two interior vertices of index 3 are adjacent. For neither could be a source or a sink, by Lemma 2.4 , and every vertex adjacent to one labelled $b a c^{-1}$ has a subword $a b^{-1}$ or $d$ in its label (see Figure 26).

Give each corner of each vertex of index $k$ an angle of $2 \pi / k$. Then by Lemma 2.5 we can find a positively curved interior face $F$, say, whence some vertex $Z$ of $F$ has index 3 .

If $b a=c$ in $G$ then any interior vertex of index less than 6 having a $d$ in its label is a sink. To see this assertion, note that $a, b$, and $c$ generate a cyclic subgroup of $G$, so any such label would have to contain at least two occurrences of $d$, for otherwise $G$ itself would be cyclic. Similarly $G$ would be cyclic if the label had the form $d^{n} b c^{-1}, d^{n} b a b^{-1}$, or $d^{n} c a c^{-1}$ (up to cyclic permutation and inversion) for some integer $n$. This rules out all possible labels except $d^{\mp 2} b a c^{-1}$ or $d^{\mp 2} c a b^{-1}$. But the first of those would imply $d^{2}=1$ and the second would imply that $G$ was abelian-by-finite, a contradiction.

If $Z$ has label $a^{3}$ and $b a=c$, then, since each vertex adjacent to $Z$ has a $d$ in its label, $F$ cannot have positive curvature by the above assertion.

If $Z$ has label $b a c^{-1}$, then at least one of the vertices adjacent to $Z$ in $F$ has a label involving $d$, and so index 6 . (This applies to both vertices adjacent to $Z$ if $Z$ is the $a$-corner of $F$.) Hence some other vertex of $F$ has index 3 . Necessarily this is the vertex $W$ opposite to $Z$ in $F$, and necessarily $Z, W$ are the $b$-, $d$-corners of $F$ respectively. Thus $W$ has label $d^{3}$ (see Figure 27).

The vertex $U$ in Figure 27 has index at least 6 , so $V$ must have index 4 or 5 , with label beginning $c a b^{-1}$. Since $d^{3}=b=a c^{-1}=1$, the only possible label which will not force $G$ to be cyclic is $c a b^{-1} c b^{-1}$. Hence $V$ has index 5 , so $U$ has index 6 or 7 , with label beginning $d c a$. Again there is only one 


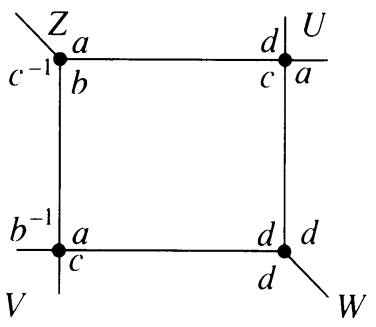

FIGURE 27

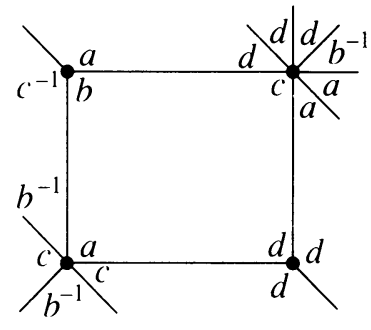

FIGURE 28

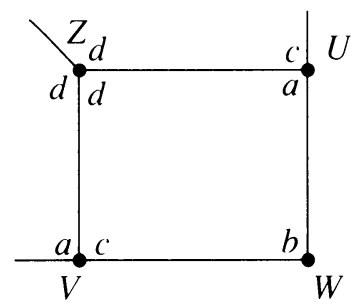

FIGURE 29

possible label which does not force $G$ to be cyclic, namely $d c a^{2} b^{-1} d^{2}$. Thus $F$ is as shown in Figure 28, and has curvature $+2 \pi / 105$.

Next suppose that $Z$ has label $d^{3}$ (Figure 29). Then $d^{3}=1$ in $G$. If the vertex $W$ in Figure 29 has index 3, then we can argue as above (after relabelling the vertices of $F$ ) to show that $F$ has the form shown in Figure 28. Hence we shall assume that $W$ has index at least 4. Consider the vertices $U$ and $V$ in Figure 29. At least one of these has index 4, so there is a relator in $G$ of length 4 beginning $c a$. Since $c a \neq d b$ in $G$ by Remark 2.3 , there are only four possibilities:

$$
c a c^{-1} d, \quad c a c^{-1} d^{-1}, \quad c a b^{-1} d, \quad c a^{2} b^{-1} .
$$

Moreover, only one of these can be a relation in $G$, for any two of them, together with the relations $b=1=d^{3}$, would imply that $G$ is cyclic.

Suppose that $b a=c$ in $G$. Then the list of labels given above reduces to $c a^{2} b^{-1}$, for otherwise we could obtain a contradiction by showing $G$ to be cyclic. If $U$ in Figure 29 has index 4, then $d$ occurs in the label of $W$ and so $W$ has index at least 6 , a contradiction. Hence $U$ has index 5 , and both $V$ and $W$ have index 4 . This forces the label of $W$ to begin $b a^{-1}$, and the only possible label for $W$ is $b a^{-2} c^{-1}$, so the label of $U$ begins $c a b^{-1}$. The only possible label for $U$ is $c a b^{-1} c b^{-1}$. Hence $F$ has the configuration shown in Figure 30, and curvature $+\pi / 15$.

Now suppose that $b a \neq c$ in $G$. We again turn our attention to Figure 29 .

Suppose first that $V$ has index 4. If its label is $\mathrm{cac}^{-1} d$, then $W$ has a label of length 4 or 5 beginning $b a$; that is, one of $b a b^{-1} d^{\mp 1}, b a c^{-1} d^{\mp 1}, b a^{2} c^{-1}$, $b a b^{-1} d^{\mp 2}, b a^{2} b^{-1} d^{\mp 1}, b a c^{-1} d^{\mp 2}, b a^{2} c^{-1} d^{\mp 1}, b a^{3} c^{-1}$, or $b a c^{-1} b c^{-1}$. But 


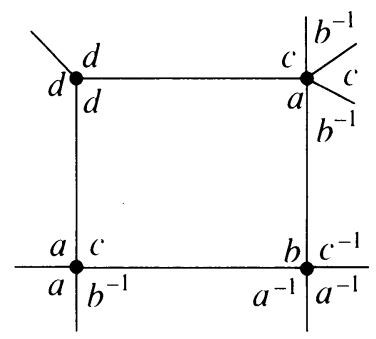

FiguRE 30

any one of these, together with the relations $b=d^{3}=c a c^{-1} d=1$, would imply that $G$ is abelian-by-finite, a contradiction.

If $V$ has label $c a b^{-1} d$, then $W$ again has one of the above labels. Any of these, together with the relations $b=d^{3}=c a b^{-1} d=1$, would imply that $G$ is cyclic or that $a^{2}=1$, with the unique exception of $b a^{2} c^{-1} d^{-1}$. But if $W$ has label $b a^{2} c^{-1} d^{-1}$, then $U$ cannot have label $c a b^{-1} d$, so must have index greater than 4 .

If $V$ has label $c a c^{-1} d^{-1}$, then $W$ has label of length 4 or 5 beginning $b c^{-1}$. The possibilities are $b c^{-1} b c^{-1}, b c^{-1} b c^{-1} d^{\mp 1}, b c^{-1} a^{\mp 1} b c^{-1}, b c^{-1} d^{\mp 2}$, $b c^{-1} d^{\mp 3}$. Any one of these other than the first, as a relation in $G$ together with $b=d^{3}=c a c^{-1} d=1$, would imply that $G$ is cyclic. But if $W$ has label $b c^{-1} b c^{-1}$, then $U$ has label of length 5 beginning $c a b^{-1}$. The only possibilities are $c a b^{-1} d^{\mp 2}$ and $c a b^{-1} c b^{-1}$, either one of which, as a relation in $G$ together with the labels of $U, W$, and $Z$ and the relation $b=1$, implies that $G$ is cyclic.

If $V$ has label $c a^{2} b^{-1}$, then $W$ has label of length 4 or 5 beginning $b a^{-1}$. This label cannot involve $d$, or $G$ would be cyclic, so the only possibilities are $b a^{-2} c^{-1}, b a^{-3} c^{-1}$, or $b a^{-1} c^{-1} b c^{-1}$. Together with the relations $b=d^{3}=$ $c a^{2} b^{-1}=1$, the second of these would imply $a=1$ and the third would imply $b a c^{-1}=1$, a contradiction. Hence the only possibility for $W$ is $b a^{-2} c^{-1}$. But then $U$ has a label of length 5 beginning $c a b^{-1}$. Again, the only possibilities are $c a b^{-1} c b^{-1}$, which contradicts $b a \neq c$, and $c a b^{-1} d^{\mp 2}$, which implies that $G$ is cyclic.

Hence $V$ cannot have index 4. We must have both $U$ and $W$ of index 4 and $V$ of index 5. If $U$ has label $c a c^{-1} d^{\mp 1}$, then $W$ has label of length 4 beginning $d^{-1} b$; that is, one of $d^{-1} b a^{\mp 1} b^{-1}, d^{-1} b a^{\mp 1} c^{-1}$, or $d^{-1} b c^{-1} d^{-1}$. Any one of these, as a relation in $G$ together with $b=d^{3}=c a c^{-1} d^{\mp 1}=1$, implies that $G$ is abelian-by-finite, a contradiction.

If $U$ has label $c a^{2} b^{-1}$, then $W$ has a label of length 4 beginning $d b$, that is one of $d b a^{\mp 1} b^{-1}, d b a^{\mp 1} c^{-1}$, or $d b c^{-1} d$. But any one of these, as a relation in $G$ together with $b=d^{3}=c a^{2} b^{-1}=1$, implies that $G$ is cyclic, a contradiction.

Hence $U$ has label $c a b^{-1} d$, and $W$ has label of length 4 beginning $c^{-1} b$. A label $c^{-1} b a^{\mp 2}$ would imply that $G$ was cyclic, so $W$ has label $c^{-1} b c^{-1} b$. Thus 


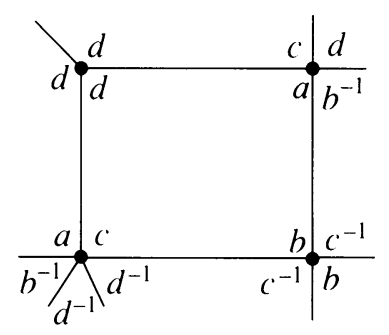

Figure 31

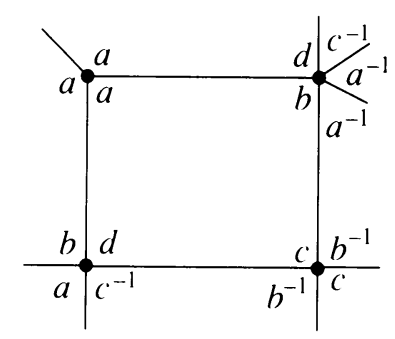

FIGURE 32

$V$ has a label of length 5 beginning $d^{-1} c a$, in other words one of $d^{-1} c a^{2} b^{-1}$, $d^{-1} c a^{2} c^{-1}, d^{-1} c a c^{-1} d^{-1}$, or $d^{-1} c a b^{-1} d^{-1}$. All but the last of these, as a relation in $G$ together with $b=d^{3}=c a b^{-1} d=1$, implies that $G$ is finite or cyclic, a contradiction.

All of this gives one further possible labelling for the positively curved face $F$, namely that shown in Figure 31 , with curvature $+\pi / 15$. In particular we have the relations $d^{3}=c a b^{-1} d=\left(c^{-1} b\right)^{2}=b=1$ in $G$. By the symmetry of the situation, there is of course one other possible labelling for the vertices of a positively curved interior face (where the vertex of index 3 is a source). This is shown in Figure 32. Note that in this case the relations we can deduce in $G$ are $a^{3}=d b c^{-1} a=\left(b^{-1} c\right)^{2}=b=1$. But the union of these two sets of relations is enough to imply that $G$ is finite, a contradiction.

Hence we may assume that all interior positively curved faces have vertices labelled as in Figure 28 or 30 , in case $b a=c$ in $G$, and as in Figure 31 in case $b a \neq c$ in $G$.

Note that in all cases the curvature of $F$ is at most $+\pi / 15$.

We say that a vertex of $D$ is good if it is the $d$-corner of an interior, positively curved face of $D$. By the proof of Lemma 2.5, the total curvature of all the interior faces is at least $+2 \pi$, so, by the note immediately above, there must be at least 30 good vertices. Our strategy is to show that the positively curved faces are at least compensated for by negatively curved faces in the neighbourhoods of the good vertices.

Let $Z$ be a good vertex having no common face with the distinguished vertex $v_{0}$. By the neighbourhood of $Z$ we mean the set of three faces incident at $Z$. 


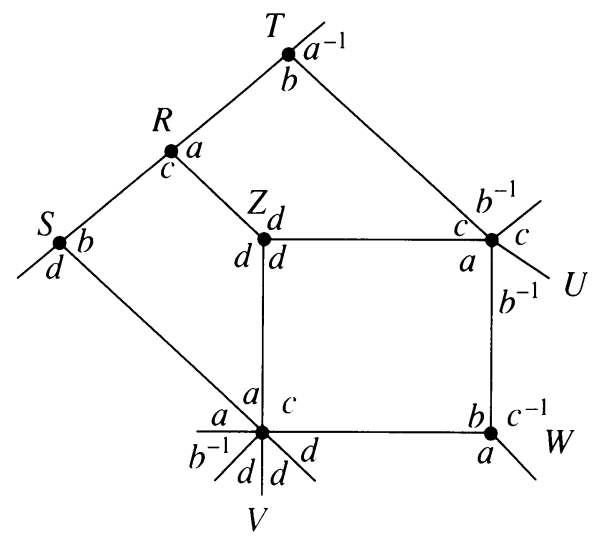

FIGURE 33

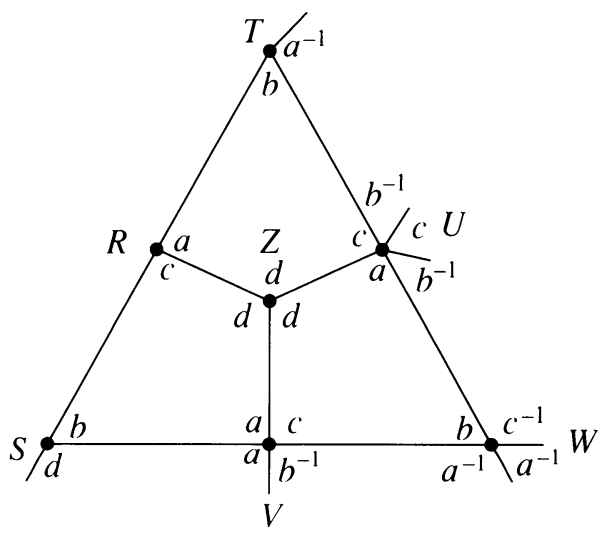

FIGURE 34

This is shown in case $b a=c$ in Figures 33 and 34, and in case $b a \neq c$ in Figure 35.

Observe in each Figure 33-35 that while $Z V W U$ is positively curved, neither $Z U T R$ nor $Z R S V$ has the correct positioning of vertex indices to be positively curved.

Indeed, in Figure $33 R$ and $S$ each have index at least 4, so $Z R S V$ has curvature at most $-\pi / 21$. Hence the total curvature of the neighbourhood of $Z$ in Figure 33 is at most $2 \pi / 105-\pi / 21=-\pi / 35$.

In Figure 34, the vertex $S$ has a $d$ in its label and so, by an earlier observation, has index at least 6. Hence if $R$ has index greater than 4, then $Z R S V$ has curvature at most $-\pi / 10$. On the other hand, if $R$ has index 4, then its label is necessarily $c a^{2} b^{-1}$, and $T$ has a $d$ in its label. It follows that $T$ has index at least 6 , and so $Z U T R$ has curvature at most $-\pi / 10$. Thus the total curvature of the neighbourhood of $Z$ in Figure 34 is at most $\pi / 15-\pi / 10=-\pi / 30$. 


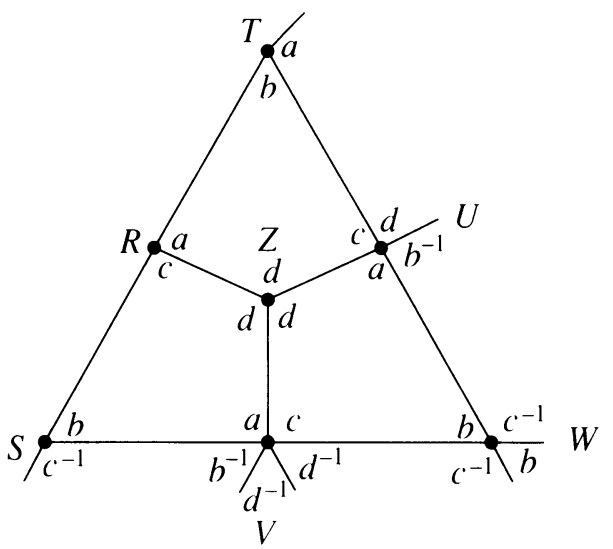

FIGURE 35

Now consider Figure 35. If $R$ has index 6 or more, then $Z R S V$ has curvature at most $-\pi / 10$. If $R$ has index 5 , then $T$ has index at least 5 , and each of ZUTR, ZRSV has curvature at most $-\pi / 30$. Finally, if $R$ has index 4 , then its label must be $c a b^{-1} d$, and $S$ has a label beginning $c^{-1} b a$. If this label has length 5 then it is either $c^{-1} b a c^{-1} b$, which would imply $a=1$, or $c^{-1} b a^{3}$, which would imply $a^{6}=1$, and $G$ is abelian-by-finite. Either of these is a contradiction, so $S$ has index at least 6 , and $Z R S V$ has curvature at most $-\pi / 10$. In all cases, the neighbourhood of $Z$ in Figure 35 has nonpositive total curvature.

It follows that most of the positive curvature of $D$ is "concentrated" near $v_{0}$. In particular, by the proof of Lemma 2.5 again, there must be at least 30 good vertices to which the above argument does not apply, in other words, which share a face with $v_{0}$. Since at most one corner of any face can be a good vertex, it follows that $v_{0}$ has index $k \geq l \geq 30$, where $l$ is the number of good vertices having a common face with $v_{0}$.

Consider the following collections of faces of $D$ :

(A) the $k$ faces incident at $v_{0}$;

(B) the $l$ positively curved interior faces whose $d$-corner has a face in common with $v_{0}$.

We can divide the remainder of $D$ into neighbourhoods of good vertices (each having nonpositive curvature), and individual nonpositively curved faces. Hence the total curvature of $(A)$ and $(B)$ is at least $+4 \pi$.

On the other hand, each face in (A) has one vertex of index at least 30 , at most two vertices of index 3 (by an earlier observation), and any other vertices have index at least 4 . Hence the curvature of any face in (A) is at most

$$
\begin{aligned}
2 \pi / k+4 \pi / 3+\pi / 2-2 \pi & =2 \pi / k-\pi / 6 \\
& \leq \pi / 15-\pi / 6 \quad(\text { since } k \geq 30) \\
& =-\pi / 10 .
\end{aligned}
$$


Since each face in (B) has a curvature at most $+\pi / 15$, the total curvature of (A) and (B) is at most

$$
l \pi / 15-k \pi / 10 \leq k \pi(1 / 15-1 / 10)=-k \pi / 30 \leq-\pi,
$$

a contradiction. This final contradiction completes the proof of the theorem.

\section{REFERENCES}

1. D. J. Collins and J. Huebschmann, Spherical diagrams and identities among relations, Math. Ann. 261 (1982), 155-183.

2. M. Edjvet, Solutions of certain sets of equations over groups, St. Andrews 1990, London Math. Soc. Lecture Notes Series, Cambridge Univ. Press (to appear).

3. M. Gerstenhaber and O. S. Rothaus, The solution of sets of equations in groups, Proc. Nat. Acad. Sci. U.S.A. 48 (1962), 1531-1533.

4. J. Howie, The solution of length three equations over groups, Proc. Edinburgh Math. Soc. (2) 26 (1983), 89-96.

5. F. Levin, Solutions of equations over groups, Bull. Amer. Math. Soc. 68 (1962), 603-604.

6. R. C. Lyndon, Equations in groups, Bol. Soc. Brasil. Mat. 11 (1980), 79-102.

7. __ Equations over cyclic groups, Preprint, Université Paris VII, 1981.

8. __ Problems in combinatorial group theory, Combinatorial Group Theory and Topology (S. M. Gersten and J. R. Stallings, eds.), Ann. of Math. Studies, no. 111, Princeton Univ. Press, Princeton, N.J., 1987, pp. 3-33.

9. B. H. Neumann, Adjunction of elements to groups, J. London Math. Soc. 18 (1942), 4-11.

10. O. S. Rothaus, On the nontriviality of some group extensions given by generators and relations, Ann. of Math. (2) 106 (1977), 559-612.

Department of Mathematics, University of Nottingham, University Park, NottingHAM NG7 2RD, ENGLAND

Department of Mathematics, Heriot-Watt University, Riccarton, Edinburgh EH14 4AS, SCOTLAND 